\title{
Une étude générale de l'assainissement pluvial urbain $*$
}

PAR

\section{Desbordes}

Maître-Assistant - Iaboratoire drydrologie et d'Aménagement des Eaux Université des Sciences et Techniques du Languedoc

Montpellier

La croissance de l'urbanisation rend de plus en plus délicat le problème de l'évacuation des eaux de toutes natures hors des grands centres urbains. Le Laboratoire d'Hydrologie et d'Aménagement des Eaux de l'Université des Sciences et Techniques du Languedoc (Montpellier) [1] étudie, depuis 1972, les types de réseaux d'assainissement les mieux adaptés à ce problème et des méthodes de calcul compatibles avec son degré de complexité et son importance économique.

\section{1 - Urbanisation et assainissement}

L'urbanisation accélérée des agglomérations peut conduire localement à d'importantes modifications des données physiques et économiques du problème de l'assainissement, justifiant une révision des conceptions en matière de réseau.

\section{1 - MODIFICATION DES DONNÉES PHYSIQUES}

L'imperméabilisation des sols, première conséquence de l'urbanisation, se traduit par une brutale augmentation des débits de pointe et des volumes du ruissellement d'origine pluviale. Ainsi, les débits de pointe par unité de surface, en provenance d'une zone entièrement urbanisée, pelivent-ils être dix à vingt fois supérieurs à ceux d'une zone rurale [2], les volumes spécifiques ruisselés étant dans des proportions encore plus considérables.

La croissance de la population, l'élévation de son niveau de confort, l'industrialisation, entraînent parallèlement une augmentation des débits et des volumes des eaux usées, donc des dimensions et du prix des stations d'épuration qu'il devient de plus en plus nécessaire de construire, en raison des nuisances diverses qui accompagnent cette évolution.

Aux nuisances des eaux usées, s'ajoute en outre une pollution des eaux de ruissellement provenant du lessivage des sols et des toitures, après une période de sécheresse. Cette pollution est concentrée brutalement dans les eaux de ruissellement au début des averses. Elle serait très élevée dans les grands centres urbains industrialisés (hydrocarbures, métaux lourds, etc.) [3].

\section{2 - MODIFICATION DES DONNÉES ÉCONOMIQUES}

Elles sont pour une bonne part la conséquence des modifications des données physiques du problème.

L'évacuation de quantités croissantes d'eaux de toutes natures entraîne une augmentation très importante des investissements consacrés à la réalisation des réseaux neufs. En outre, nombre de réseaux anciens doivent être renforcés dans des conditions souvent difficiles et coûteuses, pour faire face aux situations nées de conséquences de l'urbanisation, mal définies lors des projets. Ainsi consacre-t-on en France de 100 à 200 millions de francs par an pour l'extension et la création des réseaux d'assainissement. Ces investissements auraient en outre un taux de progression moyen annuel de l'ordre de $15 \%$ [4].

Le niveau de pollution actuelle des cours d'eau naturels rend nécessaire la construction de stations d'épuration plus importantes et plus efficaces, donc d'un prix de revient plus élevé. Il nécessite un contrôle des rejets, notamment au niveau des déversoirs d'orage des réseaux unitaires;

* Le texte intégral de cette étude (244 pages) pent être fourni par La Houlle Blanche en reproduction Xérox (prix : 249 F). 
il peut conduire, pour ces derniers, à la mise en place de dispositifs spéciaux coûteux (vannes télécommandées, stations de relèvement...) destinés à limiter les effets de la pollution des eaux de ruissellement ou la fréquence de fonctionnement du déversoir.

Enfin, une certaine conception de la notion de confort joue en faveur d'une diminution des risques de défaillance des ouvrages d'assainissement, et par là même en faveur d'une augmentation des investissements qui leur sont consacrés, indépendamment des conséquences de l'urbanisation. Ces dernières vont d'ailleurs dans le même sens, dans la mesure où l'industrialisation et l'élévation du niveau de confort se traduisent par un accroissement de la valeur des paramètres économiques d'occupation des sols.

\section{3 - RECHERCHE DE SOLUTIONS}

La croissance des nuisances engendrées par l'urbanisation et les difficultés d'adaptation des systèmes classiques à ce problème devrait, à notre avis, orienter le choix des responsables de l'aménagement urbain, vers des réseaux séparatifs d'un type particulier: les réseaux séparatifs avec réservoirs d'accumulation des eaux pluviales [5].

Le réseau d'eaux usées serait calculé à partir d'une évolution probable des consommations d'eau. Il serait muni de stations d'épuration construites progressivement. Ces stations pourraient avoir des rôles spécifiques suivant la nature des effluents à traiter (domestiques ou industriels).

Le réseau d'eaux pluviales serait calculé à partir d'une estimation de l'imperméabilisation des sols et comprendrait des bassins de régulation des écoulements orageux. Ces bassins permettent un fonctionnement plus rationnel des réseaux séparatifs, en augmentant la durée d'évacuation du ruissellement à la capacité maximale des conduites (les réseaux actuels sont en effet calculés théoriquement pour une évacuation à capacité maximale, une fois tous Ies dix ans). Ils peuvent résoudre localement des problèmes délicats de renforcement de réseaux, problèmes en général coûteux. Ils contribuent à combattre la pollution du ruissellement pluvial: ils limitent les effets d'une concentration brutale en polluants au début des averses et autorisent un traitement éventuel des eaux accumulées si l'exutoire naturel nécessite une protection particulière. Enfin, ces bassins peuvent jouer un rôle dans l'aménagement du tissu urbain. Etablis en plans d'eau naturels ou artificiels, ils peuvent valoriser certains espaces de repos. Dans ce dernier cas, il convient de maîtriser la pollution du ruissellement pluvial.

Ce type particulier de réseau présente en outre des avantages économiques. Si l'on exclut le prix des stations d'épuration, près de $80 \%$ du prix des réseaux actuels va à l'évacuation temporaire des eaux pluviales. D'une manière générale, le réseau séparatif permet un meilleur calcul du réseau d'eaux usées et des stations d'épuration dont il autorise en outre un meilleur fonctionnement. Les bassins de régulation conduisent à des économies importantes, pour un même degré de protection, par réduction des dimensions du réseau à l'aval de ces ouvrages. Certains auteurs [6] pensent ainsi que les réseaux séparatifs avec bassins d'accumulation peuvent être, au plan des frais de premier établissement, plus économiques que les réseaux unitaires, indépendamment de leurs autres avantages dont l'évaluation économique est plus délicate. En outre, une averse orageuse qui occasionnait des dégâts particuliers avec un réseau unitaire ou séparatif classique, donnera lieu à des dégâts moindres par amortissement des débits maximaux dans les réservoirs.

Cependant, le calcul de ce type de réseau ne saurait être conduit de façon précise à l'aide de la méthode actuellement en usage et consignée dans l'Instruction Technique CG 1333 (1949). Dérivée de la méthode rationnelle américaine et adaptée par $\mathrm{M}$. Caquot, elle ne permet en effet que la connaissance du débit maximal de fréquence donnée à l'exutoire d'un bassin versant; le calcul d'un réseau avec bassins de régulation nécessite la connaissance de l'hydrogramme d'écoulement en tout point du réseau, et résultant d'un hyétogramme donné. Aussi nous attachons-nous, depuis 1972, à mettre au point de nouvelles méthodes de calcul fondées sur une meilleure connaissance des phénomènes hydrologiques urbains. La complexité des processus hydrologiques a orienté notre recherche vers une modélisation mathématique de type conceptuelle. La principale difficulté réside dans le fait que les réseaux devraient être calculés économiquement en termes de risque de défaillance en raison de la nature aléatoire des précipitations. Cependant, les premiers résultats de notre recherche semblent indiquer l'efficacité de nos tentatives de modélisation générale du ruissellement urbain. Ils laissent entrevoir les possibilités d'élaboration de méthodes de calcul des réseaux mieux adaptées aux conditions d'évolution de l'urbanisation. Nous résumons ces résultats ci-après.

\section{2 - Etude de la validité du modèle de M. Caquot}

Dans un premier temps, nous avons étudié la validité du modèle de Caquot et procédé à un nouvel ajustement des valeurs numériques des paramètres de ce modèle. Nous avons vérifié expérimentalement la qualité de cet ajustement.

\section{1 - FORMULATION DU MODËLE}

La conservation des volumes d'eau mis en jeu dans le ruissellement durant l'intervalle de temps séparant le début de l'averse et l'instant $\theta$ où l'on observe le débit maximum à l'exutoire du bassin s'écrit:

$$
1 / 6 \alpha H A C=\left[\delta\left(t_{1}+t_{2}\right)+\beta \theta\right] Q_{p}
$$

$H=$ hauteur de pluie en $\mathrm{mm}$ tombée entre les instants 0 et $\theta$ à l'épicentre de l'orage;

$A=$ surface du bassin en hectares;

$C=$ coefficient de ruissellement $(H C=$ volume de «pluie nette $\gg$ );

$\alpha=$ coefficient traduisant la distribution spatiale de l'averse $=A^{-\varepsilon}$,

$t_{1}=$ temps de parcours de l'eau dans le réseau, en minutes;

$t_{2}=$ temps de parcours de l'eau sur le sol, en minutes;

$t_{1}+t_{2}=$ «temps de concentration » du bas$\sin =t_{c}$;

$Q_{p}=$ débit de pointe en $\mathrm{m}^{3} / \mathrm{s}$

$\delta\left(t_{1}+t_{2}\right) Q_{p}=$ volume d'eau stocké sur le sol et dans le réseau entre 0 et $\theta$ minutes;

$\beta \theta Q_{p}=$ volume d'eau écoulé à l'exutoire entre 0 et $\theta$. 
L'équation (1) devient pour $t_{1}+t_{2}=\theta=t_{c}$ (majoration de $Q_{p}$ si $\left.\left(t_{1}+t_{2}\right)>\theta\right)$

$$
\frac{1}{6(\beta+\delta)} \alpha C A \frac{H}{t_{c}}=Q_{p}
$$

$H / t_{c}$ représente l'intensité moyenne sur la durée $t_{c}$. Or, pour une période de retour donnée $T$ (inverse de la probabilité de dépassement), l'intensité maximale moyenne sur toute durée $t$ peut régionalement être représentée de façon approximative par la relation:

$$
i(t, T)=a(T) t^{b(T)}
$$

L'équation (2) peut alors s'écrire avec $\alpha=A^{-\varepsilon}$ :

$$
\begin{aligned}
Q_{p}(T) & =\frac{1}{6(\beta+\delta)} \alpha C A i_{\text {max }}\left(t_{c}, T\right) \\
Q_{p}(T) & =\frac{1}{6(\beta+\delta)} C a(T) t_{c}^{b(T)} A^{1-\varepsilon}
\end{aligned}
$$

A partir de considérations théoriques sur les temps d'écoulement, M. Caquot a trouvé une expression du temps de concentration sous la forme:

$$
t_{0}=\mu I^{o} A^{d} Q_{p}^{j}=t_{1}+t_{2}
$$

où $\mu$ est un coefficient numérique d'ajustement, $I$ pente moyenne du bassin en mètre par mètre.

La combinaison des équations (5) et (6) donne:

$$
Q_{p}(T)=\left[\frac{a \mu^{b}}{6(\beta+\delta)}\right]^{1 /(1-b f)} \times I^{(b l(1-b f)} \times C^{1 /(1-b f)}
$$

Ainsi, lorsque les valeurs numériques des neuf paramètres $a, b, c, d, f, \mu, \beta, \delta, \varepsilon$ sont déterminées, le modèle de Caquot peut-il se résumer à la forme monome plus connue :

$$
Q_{p}(T)=K(T) \times I^{u(T)} \times C^{v(T)} \times A^{w(T)}
$$

L'équation (8) peut être aisément mise en abaque d'un grand intérêt pour le projeteur s'il peut connaître sans ambiguiité les paramètres physiques $I, C, A$ des bassins qu'il doit aménager.

Pour la réalisation de réseaux simples ne nécessitant que la connaissance du débit de pointe $Q_{p}$, le modèle de Caquot apparaît comme un outil efficace à la condition que les hypothèses ayant conduit à son élaboration traduisent la réalité des phénomènes et que les paramètres d'ajustement aient été correctement évalués.

\section{2 - ANALYSE DES HYPOTHÉSES DE BASE DU MODËLE}

L'équation (4) montre que ce modèle n'est en réalité qu'une adaptation de la méthode rationnelle (1851) en usage aux Etats-Unis et qui s'écrit:

$$
Q_{p}(T)=C \times i\left(t_{i}, T\right) \times A
$$

Cette méthode repose sur le concept de «temps de concentration $» t_{c}$, qui est le temps de parcours de l'eau entre l'exutoire du bassin versant et le point qui en est le plus hydrauliquement éloigné. Ce concept suppose que le débit maximum ne peut être observé, à l'exutoire, que lorsque la totalité du bassin a apporté sa contribution au débit; c'est-à-dire sous l'effet d'une averse dont la durée est au moins égale à celle du «temps de concentration» $\left[t_{1}+t_{2}=\theta\right.$ dans l'équation (2)].

Cette méthode admet en outre la linéarité de la transformation de la pluie en débit. Elle implique que la réponse $\mathrm{du}$ bassin versant, à une averse de forme quelconque et de durée $t \leqslant t_{c}$, soit identique à celle d'une averse rectangulaire, de même durée et d'intensité constante, égale à l'intensité moyenne de la précédente. Dans le modèle de M. Caquot, l'hypothèse de linéarité apparaît dans la prise en compte de paramètres $\beta$ et $\delta$ constants.

M. Caquot a cependant apporté trois modifications à «la méthode rationnelle». En premier lieu, il suppose un amortissement de l'intensité $i\left(t_{c}\right)$, lié au stockage temporaire de l'eau sur le sol et dans le réseau; l'influence de cet amortissement est traduit par les paramètres $\beta$ et $\delta$ de l'équation (4). En outre, il prend en compte les effets de la distribution spatiale des averses qui correspondraient, pour une averse stationnaire, à une décroissance de l'intensité ponctuelle, en fonction de la distance à l'épicentre. M. Caquot intègre ces effets sous la forme d'un coefficient de réduction qu'il choisit par commodité du type $A^{-\varepsilon}$ [équation (5)]. En troisième lieu, il introduit un intéressant élément de non linéarité, en supposant que «le temps de concentration » n'est pas constant et dépend du débit de pointe [équation (6)]. Son modèle est donc pseudo-linéaire (transformation linéaire pour une averse donnée mais évoluant d'une averse à l'autre).

Enfin, les deux modèles comportent une hypothèse probabiliste, fondamentale pour le calcul des ouvrages en termes de risque de défaillance, en cela qu'ils supposent que l'intensité maximale moyenne sur la durée du temps de concentration, et le débit qui en résulte, ont même probabilité d'occurrence.

Les hypothèses déterministes du modèle de Caquot sont sans doute proches de la réalité, dans la mesure où les bassins versants présentent des caractéristiques homogènes de pente et de coefficients de ruissellement. Elles doivent conduire à des ordres de grandeur corrects pour les débits, si les paramètres numériques du modèle sont convenablement estimés. Cependant, en raison de la non linéarité de la transformation de la pluie en débit, nous pensons que la valeur numérique de ces paramètres doit être principalement liée à la taille des bassins versants. Ainsi, conviendrait-il, pour chaque ajustement, de donner ses limites d'application. Les résultats de nos travaux nous incitent en outre à penser que ce modèle ne soit pas applicable aux bassins versants de grandes dimensions (hétérogénéité des caractéristiques physiques).

L'hypothèse probabiliste suppose implicitement que le coefficient de ruissellement $C$ ne soit pas une variable aléatoire. En particulier, en hydrologie rurale classique, $C$ dépend des pluies antérieures, variables aléatoires. Nos travaux [1] ont montré cependant qu'en zone urbaine, le coefficient $C$ pouvait être approché par le rapport des surfaces imperméabilisées à la surface totale, tout du moins pour un domaine expérimental limité inférieurement à $20 \%$ dimperméabilisation. Dans ces conditions, le coefficient $C$ apparaît, en hydrologie urbaine, comme une variable déterministe jouant en faveur de l'hypothèse probabiliste du modèle de M. Caquot. 


\section{3 - AJUSTEMENT NUMERIQUE ET DOMAINE DE VALIDITÉ}

Nous avons procédé à l'estimation des paramètres $\mu, c$, $d, f, \beta, \delta$ et $\varepsilon$ du modèle. Dans l'Instruction CG 1333, ces valeurs sont :

$$
\begin{gathered}
\mu=0,93 ; \quad C=-0,363 ; \quad d=0,366 \\
f=-0,2 ; \quad \beta+\delta=1,5 ; \quad \varepsilon=0,1 .
\end{gathered}
$$

Reprenant les travaux de Izzard [7], Hicks [8], Barnes [9] sur le ruissellement superficiel et les écoulements en conduite, ainsi que les résultats de nombreuses expériences réalisées principalement aux Etats-Unis, nous avons donné une nouvelle expression du «temps de concentration» des bassins versants, soit:

$$
\begin{gathered}
t_{c}(\mathrm{mn})=0,65 \times I^{-0,41}(\mathrm{~m} / \mathrm{m}) \times A^{0,51}(\mathrm{ha}) \\
\times Q_{j}{ }^{-0,29}\left(\mathrm{~m}^{3} / \mathrm{s}\right)
\end{gathered}
$$

La relation (10) revient à majorer de façon importante (20 à $80 \%$ suivant les cas) la durée de $t_{t}$ donnée par M. Caquot, durée qui conduit en général à des vitesses supérieures à la réalité [9].

En analysant des hydrogrammes simples observés sur des petits bassins versants expérimentaux gérés par SO.GRE.A.H. [10] nous avons constaté que les paramètres $\beta$ et $\delta$ variaient notablement pour un même bassin (non linéarité) et que leur somme était en général très inférieure à la valeur de 1,5 donnée par la circulaire. Nous avons retenu la valeur moyenne des observations, soit:

$$
\beta+\delta=1,1
$$

Nous pensons cependant que la valeur de $\beta+\delta$ doit augmenter avec la taille des bassins versants.

L'examen de données expérimentales [11] récentes, nous a amenés à conclure que la valeur $\varepsilon=0,1$ était trop réductrice. A cela, il convient d'ajouter que le déplacement des averses orageuses sur le bassin peut avoir, dans certains cas, un effet sur les débits de pointe comparable à celui qui résulterait dans l'expression (4), d'une majoration de l'intensité maximale au centre de l'averse. En outre, l'information pluviométrique dont nous disposons, et qui est utilisée pour le calcul des coefficients $a$ et $b$ de la relation (3), est tellement dispersée qu'elle a une probabilité négligeable de représenter l'intensité maximale des orages en leur centre, et qu'elle intègre implicitement les effets liés à la distribution spatiale. Par comparaison avec d'autres relations proposées par ailleurs, nous donnons en première approximation :

$$
\varepsilon=0,05
$$

Nous avons procédé [12] à une vérification de notre ajustement numérique à partir des données expérimentales recueillies par SO.GRE.A.H., en utilisant, pour un couple d'averses et de débit enregistrés, le bilan donné par l'équation (2), les valeurs numériques des divers paramètres données par les relations (10), (11), (12) et en considérant le coefficient $C$ comme égal au rapport des surfaces imperméables à la surface totale, (ce que nous avions vérifié par ailleurs [1]). Cette vérification nous a montré que notre ajustement sous-estimait légèrement les débits alors que celui de la circulaire CG 1333 les sous-estimait très fortement. Nous avons constaté que l'adéquation de notre ajustement était réalisée lorsque l'on utilisait un temps de concentration $t_{c^{\prime}}$ inférieur au temps $t_{c}$ donné par la relation $(10)$, soit :

$$
t_{c^{\prime}}=0,8 t_{c}
$$

ou encore en faisant $\mu=0,5$ dans cette même relation (10). D. Normand a étudié cette adéquation [13] et en a conclu qu'elle était correcte pour des bassins atteignant 200 hectares. Le domaine de validité de notre ajustement pour des bassins de caractéristiques homogènes serait actuellement le suivant:

$$
A<200 \text { ha } \quad ; \quad 0,2 \%<I<5 \% \quad ; \quad 0,2<C<1
$$

Dans le cas d'association de bassins de caractéristiques physiques très hétérogènes, le modèle de Caquot, beaucoup trop global, ne peut conduire qu'à des résultats très approximatifs voire erronés. Seule une analyse plus fine des phénomènes hydrologiques permettra de lever cette difficulté.

\section{3 - Modélisation des phénomènes hydrologiques urbains}

Le modèle de Caquot semble limité aux bassins homogènes de petite superficie. En outre, il ne permet que le calcul d'un seul point de l'hydrogramme: le débit de pointe. Pour faire face à ces insuffisances, nous étudions de nouvelles méthodes de calcul fondées sur une modélisation générale des phénomènes hydrologiques urbains, rencontrés au cours des étapes du calcul d'un réseau. Les différents modèles à mettre en cuvre sont les suivants:

- Modèle stochastique de la «pluie brute», entrée du système, constitué par le bassin et son réseau. Ce modèle devrait servir à définir «la pluie de projet» autorisant le calcul du réseau en termes de risque. (Dans le cas du modèle de ruissellement de $M$. Caquot, ce modèle de pluie est constitué par les classiques courbes «intensité-durée-fréquence ».)

- Modèle de pertes au ruissellement permettant de passer de la «pluie brute» à la «pluie nette» ruisselée. Ces pertes peuvent varier dans le temps et l'espace.

- Modèle hydrologique élémentaire permettant de connaître l'hydrogramme, résultant d'une pluie donnée, à l'exutoire d'un bassin versant équipé d'un réseau de collecteurs secondaires. Ce modèle serait appliqué aux sous-bassins homogènes de faible superficie (quelques dizaines d'hectares) d'une unité hydrologique importante dont on veut calculer ou étudier le réseau.

- Modèle hydraulique de propagation des hydrogrammes élémentaires des sous-bassins dans les collecteurs principaux de l'unité hydrologique.

- Modèle économique permettant de réaliser un compromis entre les investissements et les dégâts résultant de la défaillance des ouvrages d'assainissement.

Nous ne traiterons ci-après que des modèles strictement hydrologiques. Les modèles hydrauliques sont d'ores et déjà fort nombreux et il suffirait actuellement de ne retenir que le plus simple, tout en étant en accord avec le degré de précision nécessaire dans un projet de réseau. Quant à la modélisation économique, nous pensons qu'il s'agit là d'un problème sans difficulté du point de vue théorique, et ne 
nécessitant pas la recherche d'une méthodologie nouvelle. Par contre, l'évaluation monétaire des dégâts est délicate car l'information dans ce domaine est actuellement très réduite.

\section{1 - ÉTUde des PERTES AU RUISSEllement}

L'analyse de données expérimentales nous a conduits à conclure, pour l'instant, à la prépondérance des surfaces imperméabilisées dans le ruissellement urbain, au point que l'on puisse négliger l'apport des surfaces perméables dès que le pourcentage des premières, $I M P$, est supérieur à $20 \%$.

La nécessité d'une modélisation des pertes au ruissellement n'a pu donc être mise en évidence par l'expérimentation actuelle. Cependant nous pensons que, sous des averses violentes et pour des terrains peu perméables à forte pente, la réponse de ces terrains peut ne plus être négligeable devant celle des surfaces imperméables. Une étude expérimentale reste donc nécessaire pour résoudre ce problème. Il peut être localement d'importance, lorsque la zone urbaine se trouve, par exemple, à l'aval d'un bassin rural de grande superficie, drainé plus ou moins partiellement par le réseau urbain.

Notre étude a cependant permis de donner une signification physique au coefficient $C$ du modèle de Caquot. En le reliant à $I M P$, il est alors possible de prendre en compte, dans les projets, les effets liés à diverses hypothèses d'urbanisation de la zone à assainir. Il suffirait pour cela d'établir des corrélations entre l'imperméabilisation des sols et leur mode d'occupation. Les services du Ministère de l'Equipement étudient d'ailleurs ce problème.

\section{2 - MODẼLE HYDROLOGIQUE ÉLÉMENTAIRE}

La complexité relative des processus physiques traduisant la transformation de la pluie en débit, à l'exutoire d'un petit bassin versant urbain équipé d'un réseau de drainage, et surtout les difficultés d'évaluation des paramètres hydrauliques intervenant dans cette transformation, nous ont orientés vers un modèle de type conceptuel, dérivé de l'analyse des systèmes. Le bassin versant est alors considéré comme un système de transformation dont la pluie représente l'entrée, et le débit la sortie.

Nous avons analysé les divers modèles conceptuels linéaires utilisables en hydrologie urbaine [1]. L'allure générale des hydrogrammes expérimentaux dont nous disposions, nous a conduits à retenir un modèle traduisant un effet de stockage. Un tel modèle résulte de l'association de l'équation de conservation des volumes entrant et sortant du système, et d'une équation de stockage proprement dite dont la forme générale peut s'écrire:

$$
S(t)=\sum_{i=0}^{k=n} A_{k}(i, Q, t) \frac{d^{l i} Q}{d t^{\lambda_{i}}}+\sum_{i=0}^{j=m} B_{j}(i, Q, t) \frac{d i_{i}}{d t^{j}}
$$

$S(t)$ représente le stockage, à l'instant $t$, dans le système; $i$ la pluie et $Q$ le débit. Afin que le modèle puisse être de quelque utilité dans un projet de réseau, c'est-à-dire dans le cas de bassins versants pour lesquels on ne dispose pas de mesure débitmétrique, nous avons retenu une équation très simple comportant un nombre minimum de paramètres, soit :

$$
S(t)=K Q(t)
$$

associée à l'équation de continuité,

$$
d S(t) / d t=i(t)-Q(t)
$$

L'équation (15) conduit à l'équation différentielle du processus de transformation de la pluie en débit dont la solution générale est:

$$
Q(t)=Q_{0} e^{-\left(t-t_{0}\right) / K}+1 / K \int_{t_{0}}^{t} i(u) e^{-(t-u) / K} d u
$$

Pour un réseau séparatif vide au début de l'averse, $t_{0}=0$ et $Q_{0}=0$. La réponse impulsionnelle du modèle, appelée parfois hydrogramme unitaire instantané, est alors:

$$
h(t)=(1 / K) e^{-t / K}
$$

Le paramètre $K$ du modèle, homogène à un temps, correspond au décalage entre les centres de gravité de la pluie nette et du débit.

L'entrée $i(t)$ n'étant pas connue sous forme analytique, l'intégration numérique de l'équation (17) sous la condition initiale d'un réseau séparatif vide peut être réalisée à un pas de temps $d t$ par la relation récurrente suivante:

$$
Q(n d t)=e^{-1 / K} Q[(n-1) d t]+\left(1-e^{-1 / K}\right) i(n d t)
$$

Dans la relation (19) le paramètre $K$ doit être exprimé en unités de pas de temps d'intégration.

Calculant la valeur observée du paramètre $K$ à partir de sa définition physique de décalage et utilisant l'équation (19), nous avons tenté de reproduire 55 hydrogrammes observés sur les bassins expérimentaux français [10]. Nous avons constaté que, pour un même bassin, le paramètre $K$ variait largement d'une averse à l'autre, et que sa valeur devait être réduite systématiquement de 5 à $20 \%$, pour obtenir un écart entre les débits de pointe calculés et observés inférieurs à $5 \%$ (la relation (19) conserve par contre les volumes quel que soit $K$ ).

La variation du paramètre $K$, d'une averse à l'autre, nous a incité à chercher une liaison entre ce paramètre et les caractéristiques physiques des averses. Nous avons alors établi par corrélations multiples, pour 13 bassins expérimentaux [10] [14], la corrélation suivante :

$$
\begin{array}{r}
K=5,07 \times A^{0,18} \times p^{-0,36} \times[1+(I M P / 100)]^{-1,9} \\
\times T E^{0,21} \times L^{0,15} \times H P E^{-0,07}
\end{array}
$$

(coefficient de corrélation multiple 0,95 )

Dans la relation (20), $K$ est en minutes, $A$ est la surface du bassin en hectares, $p$ est la pente moyenne en pourcentage, IMP est le pourcentage de surface imperméable, $T E$ la durée de l'averse en minutes, $L$ la longueur du bassin en mètres, HPE la hauteur de pluie nette en millimètres. La gamme de variation des paramètres de la relation (20) était :

$$
\begin{aligned}
0,4 \mathrm{ha} & \leqslant A \leqslant 5000 \mathrm{ha} \\
2 \% & \leqslant I M P \leqslant 100 \% \\
110 \mathrm{~m} & \leqslant L \leqslant 17800 \mathrm{~m} \\
0,4 \% & \leqslant P \leqslant 5 \% \\
5 \mathrm{mn} & \leqslant T E \leqslant 180 \mathrm{mn} \\
5 \mathrm{~mm} & \leqslant H P E \leqslant 240 \mathrm{~mm}
\end{aligned}
$$


La variation de $K$ en fonction de $H P E$ et $T E$, d'averse en averse, fait que le modèle est de type pseudo-linéaire.

Nous avons utilisé la relation (20) pour reproduire des hydrogrammes observés parmi ceux qui n'avaient pas été retenus pour établir cette relation. Ainsi près de $80 \%$ des hydrogrammes reproduits l'ont-ils été avec une erreur maximale sur le débit de pointe inférieure à $20 \%$. Les erreurs plus importantes concernaient des averses très faibles, ne présentant aucun intérêt pour le calcul des réseaux.

Le modèle que nous avons étudié présente l'avantage de pouvoir être utilisé pour des bassins non jaugés, c'est-à-dire pour la totalité des bassins que l'on désire équiper d'un réseau de drainage. Il permet, en outre, de simuler les conséquences liées à diverses hypothèses d'urbanisation. Il permet, enfin, d'obtenir l'hydrogramme complet de ruissellement résultant d'une averse de caractéristiques données, et par suite un calcul plus précis des réseaux complexes comportant notamment des bassins d'orage, des stations de relèvement, etc. Il peut être utilisé, de ce fait, dans l'amélioration et la gestion de grands réseaux existants. Ainsi certaines villes des Etats-Unis sont-elles dotées d'un système automatique de gestion par ordinateur de leur réseau d'assainissement [15]. Les informations pluviométriques et débitmétriques provenant du bassin sont transmises à l'ordinateur. Ce dernier prévoit l'évolution de la situation du réseau à partir d'une modélisation du ruissellement urbain et des écoulements dans le réseau. Il déclenche en conséquence les opérations conduisant à une utilisation maximale de la capacité du réseau.

\section{3 - VERS UNE MODÉLISATION STOCHAS- TIQUE DE LA PLUIE}

Avant de poursuivre nos recherches, nous avons étudié la sensibilité générale de la transformation pluie-débit aux paramètres du modèle et de la pluie [16].

En premier lieu, le paramètre $K$ n'est connu que de façon statistique. Si l'erreur d'échantillonnage sur $K$ devait entraîner une variation importante du temps de montée et du débit de pointe de l'hydrogramme résultant d'une averse donnée, le modèle ne présenterait aucun intérêt pour des bassins non jaugés. Ainsi nous avons calculé que, pour les combinaisons extrêmes du domaine de variation (relation 21), des paramètres de l'équation (20), on avait $85 \%$ de chances de connaître la valeur de $K$ à $40 \%$ près. Nous avons alors étudié, pour $5<K<150$ unités de pas de temps d'intégration, l'influence d'un écart $\Delta K$ sur la réponse à des averses de formes diverses et de durées très variées. Nous avons constaté qu'un écart relatif de $40 \%$ sur $K$ entraîne des variations sur le débit de pointe inférieures à $20 \%$ (soit une incertitude sur le diamètre des conduites de l'ordre de $8 \%$ ), et des variations de quelques pour cent sur le temps de montée. Le modèle est donc assez peu sensible, toutes proportions gardées, à la valeur du paramètre $K$ et présente, dans la majorité des cas, un degré de précision à notre avis suffisant pour le calcul des projets de réseaux. Dans le cas de l'amélioration de réseaux existants, pour lesquels on peut procéder à des mesures de débit, le calage du modèle, à partir des observations, permettrait bien sûr d'obtenir un degré de précision bien plus élevé.

En second lieu, nos essais de définition d'une «pluie de projet $\gg[1]$ n'ont pas été très concluants en raison du caractère très aléatoire des précipitations. Aussi nous a-t-il paru nécessaire d'étudier également la sensibilité de la transformation, opérée par le modèle, aux paramètres défnissant la pluie, afin de juger de leur importance relative et de pouvoir éventuellement retenir les plus importants pour définir la pluie de projet. Nous avons alors constitué des pluies de formes géométriques simples (triangulaire, rectangulaire, exponentielle). Chaque pluie était définie en outre par sa durée, son volume et la position dans le temps de son maximum instantané d'intensité. Volume et durée étaienr liés par le biais des courbes «intensité-durée-fréquence».

Pour une large gamme du paramètre du modèle, nous avons alors obtenu les principaux résultats suivants:

- Le modèle met en évidence l'existence d'une durée critique $B_{n}$ à laquelle il est peu sensible et toujours plus faible que le classique temps de concentration. $B_{m}$ est la durée de la pluie conduisant au débit maximum pour une valeur de $K$ et une forme d'averse donnés. Au-delà de $B=3$ à 4 heures, les variations introduites sur le débit de pointe et le temps de pointe sont négligeables. La «pluie de projet $\gg$ pourrait donc être définie par cette durée maximale.

- On constate des écarts négligeables sur la réponse du modèle entre les averses de formes triangulaires et exponentielles, malgré des variations de pointe d'intensité atteignant $50 \%$.

- La position du maximum d'intensité instantanée apparaît comme un paramètre très important, surtout en regard du temps de montée de l'hydrogramme de réponse.

- La discrétisation de l'averse à un pas de temps donné a une infuence négligeable dès que sa durée $B$ est supérieure à 6 à 8 pas de temps.

- L'introduction, sur la forme géométrique simple de l'averse, d'aléas distribués suivant une loi log-normale de coefficient de variation très élevé (atteignant jusqu'à $300 \%$ au-delà de 30 minutes de part et d'autre du maximum d'intensité) entraîne des écarts sur le débit de pointe de l'ordre de 5 à $10 \%$ maximum pour $75 \%$ des 600 simulations réalisées.

Il semblerait donc que la pluie puisse être correctement définie, de façon statistique, au moyen de trois variables aléatoires : l'intensité maximale moyenne sur 3 à 4 heures, l'intensité maximale moyenne sur une durée de 15 minutes à 1 heure représentant la période de pluie intense de l'averse, et la position de la période de pluie intense sur la durée de 4 heures. L'étude des liaisons statistiques entre ces trois paramètres et de leurs lois de distribution, devrait permettre de réaliser un modèle stochastique à trois varıables aléatoires. Ce modèle permettrait de simuler des averses sur une période donnée et d'analyser statistiquement les variables des hydrogrammes résultants (débit de pointe, volume au-dessus d'un seuil de débit, etc.). On pourrait alors donner la valeur d'un débit ou volume de période de retour donnée, indépendamment de la probabilité d'occurrence de la totalité de la pluie qui le provoque (probabilitê qui est d'ailleurs négligeable).

\section{4 - Conclusions}

Les études réalisées par le Laboratoire d'Hydrologie de Montpellier dans le domaine de l'hydrologie urbaine, montrent que la modélisation mathématique des phénomènes peut être une voie intéressante vers la recherche de nou- 
velles méthodes de calcul des réseaux d'assainissement urbains. Une expérimentation reste cependant nécessaire : elle devrait permettre de mieux définir les limites de validité du modèle dans le cas de bassins peu urbanisés, le rôle éventuel des surfaces perméables sur le ruissellement, l'importance des phénomènes de distribution spatiale des averses.

\section{Bibliographie}

[1] Desbordes (M.). - «Réflexions sur les méthodes de calcul des réseaux urbains d'assainissement pluvial». Thèse d'Ingénieur-Docteur, 224 p., Montpellier (février 1974).

[2] Moore (W. L.), Morgan (C. W.). - «Effects of Watershed changes on streamflow ». Water Resources Symposium $n^{\circ} 2$, 290 p., University of Texas, Austin (1969).

[3] Environmental Protectron Agency. - Projects of the Municipal Technology. Branch through June 1972. E.P.A.R., $n^{\circ} 2-72-080$.

[4] Affholder (M.). - Contribution d'une étude expérimentale à l'amélioration des méthodes d'évaluation des débits de ruissellement en milieu urbain. Association Française pour l'Etude des Irrigations et du. Drainage. Journée d'études de Colmar, 13 p. (1970).

[5] Desbordes (M.). - Les réservoirs d'accumulation des ealux pluviales. Construction (Dunod éd.), n⿳1, pp. 19-25 (janvier 1975).
[6] JACOBSEN (E.). - La régularisation des écoulements de pluie. Etude fréquentielle des pluies. Le Génie Rural, $\mathrm{n}^{\circ} 1$, $\mathrm{pp}$ 27-31 (janvier 1973).

[7] IzZARD (C. G.). - Hydraulic of runoff from developed surfaces. Proceedings of the 26th annual meeting. Highway Research Board, vol. 26, pp. 129-146 (1946)

[8] Hicks (W. I.), - A method of computing urban runoff. Transactions of the A.S.C.E., vol. 109, pp. 1227-1253 (1944).

[9] Barnes (A.). - Flood routing through storm drains. Colorado State University, Hydrology papers $\mathrm{n}^{\circ} 43-44-45-46$, Fort Collins (1970).

[10] Normand (D.), ReNÉ (R.). - Etude générale du ruissellement urbain; Annexes A 1 et A 2. Ministère de l'Equipement et du Logement, D.A.F.U., Rapport R. 10737 (1971).

[11] Osborn (H. B.). - Thunderstorm runoft in south-eastern Arizona. Joumal of the Hydraulics division, Vol. 99, $\mathrm{n}^{\circ} \mathrm{HY} 7$, pp. 1129-1145 (1973).

[12] Desbordes (M.), - «Vérification expérimentale du modèle de Caquot». Direction Générale des Collectivités locales. Note LHM 9/74, 31 p., Ministère de l'Intérieur (1974).

[13] Normand (D). - Ajustement expérimental de la formule de Caquot en hydrologie urbaine. La Houille Blanche, $\mathrm{n}^{\circ} 4 / 5$, pp. 357-361 (1974).

[14] Sarma (P. S.), Delleur (J. W.), Rao. - A programm in urban hydrology, Part II. Technical report $\mathrm{n}^{\circ}$ 9, Purdue University Indiana (1959).

[15] Gibbs (V. C.), Alexander (M. S.), Leiser (C. P.). - System for regulation of combined sewage flows. Journal of the Sanitary Engineering Division, pp. $951-972$ (décembre 1972).

[16] Desbordes (M.), Raous (P.). — «Etude de sensibilité du modèle de ruissellement ». Laboratoire National d'Hydraulique. Direction des Etudes et Recherches d'E.D.F., Note $\mathrm{CO} 45 / 7 / 74,68$ p. (décembre 1974). 